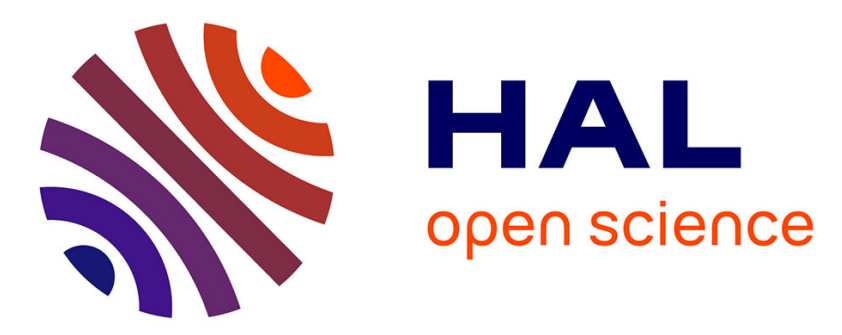

\title{
Development and validation of a new efficient SRTM DEM-based horizon model combined with optimization and error prediction methods
}

Benjamin Pillot, Marc Muselli, Philippe Poggi, Pierrick Haurant, João Batista Dias

\section{To cite this version:}

Benjamin Pillot, Marc Muselli, Philippe Poggi, Pierrick Haurant, João Batista Dias. Development and validation of a new efficient SRTM DEM-based horizon model combined with optimization and error prediction methods. Solar Energy, 2016, 129, pp.101-114. 10.1016/j.solener.2016.01.058 . hal01274911

\section{HAL Id: hal-01274911 \\ https://hal.science/hal-01274911}

Submitted on 16 Feb 2016

HAL is a multi-disciplinary open access archive for the deposit and dissemination of scientific research documents, whether they are published or not. The documents may come from teaching and research institutions in France or abroad, or from public or private research centers.
L'archive ouverte pluridisciplinaire HAL, est destinée au dépôt et à la diffusion de documents scientifiques de niveau recherche, publiés ou non, émanant des établissements d'enseignement et de recherche français ou étrangers, des laboratoires publics ou privés. 


\title{
Development and validation of a new efficient SRTM DEM-based horizon model combined with optimization and error prediction methods
}

\author{
Benjamin Pillot ${ }^{\mathrm{a}, *}$, Marc Muselli ${ }^{\mathrm{b}}$, Philippe Poggi ${ }^{\mathrm{b}}$, Pierrick Haurant $^{\mathrm{b}}$, João Batista Dias ${ }^{\mathrm{a}}$ \\ ${ }^{a}$ Universidade do Vale do Rio dos Sinos, Programa de Pós-Graduação em Engenharia Mecânica, Avenida Unisinos 950, 93022-000 São Leopoldo, Brasil \\ ${ }^{b}$ Université de Corse, UMR CNRS 6134 SPE, Route des Sanguinaires, 20000 Ajaccio, France
}

\begin{abstract}
Several post-processing methods have been developed over the last years in order to take into consideration topography within satellite-based solar radiation maps using digital elevation models (DEM). If the main part of these procedures is to estimate the obstructed horizon around each DEM point of a given region so as to consider terrain-based shading effects, the size of the area can also limit this implementation. That is why we have developed a new efficient horizon model based on the DEM retrieved during the Shuttle Radar Topography Mission (SRTM). In order to be usable at any world location with the same expected accuracy, this model is only derived from mathematical statements without any kind of empirical approximation. Validation against in situ horizons and comparison with some other models have finally shown this one presents both better accuracy (RMSE of $1.555^{\circ}$ against $1.712^{\circ}$ or more) and lower computation time (at least 4 times faster). Furthermore, in the case of very large areas, we propose an optimization procedure allowing the user to knowingly alter the modeling error in order to reduce processing time. Finally, using in situ data, we have also developed a method for predicting the repercussion of the original SRTM DEM error on the final horizon precision.
\end{abstract}

Keywords: horizon model; SRTM DEM; solar radiation maps; error prediction

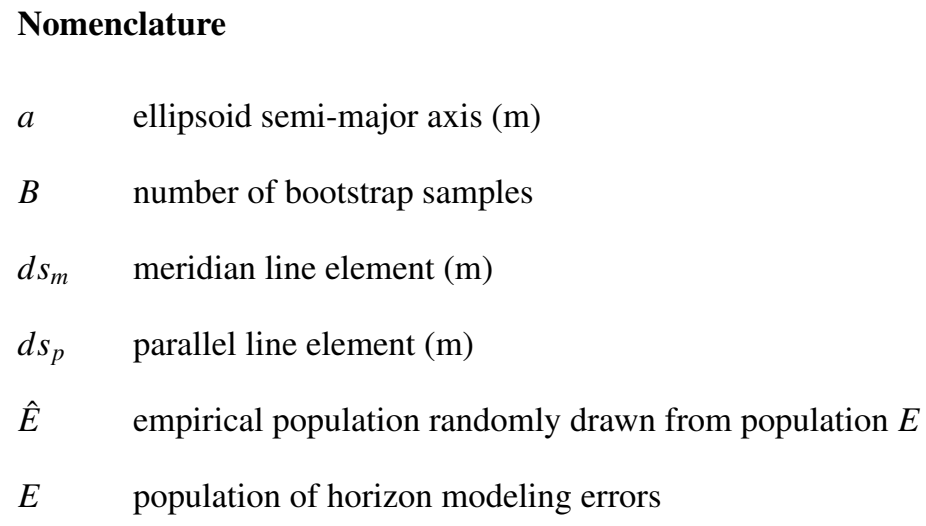

Nomenclature

$a \quad$ ellipsoid semi-major axis (m)

$B \quad$ number of bootstrap samples

$d s_{m} \quad$ meridian line element $(\mathrm{m})$

$d s_{p} \quad$ parallel line element (m)

$\hat{E} \quad$ empirical population randomly drawn from population $E$

E population of horizon modeling errors

\footnotetext{
${ }^{*}$ Corresponding author

Email addresses: benjaminfp@unisinos.br (Benjamin Pillot), marc.muselli@univ-corse.fr (Marc Muselli), philippe.poggi@univ-corse.fr (Philippe Poggi), haurant@univ-corse.fr (Pierrick Haurant), joaobd@unisinos .br (João Batista Dias)
} 


\begin{tabular}{|c|c|}
\hline$e$ & ellipsoid eccentricity \\
\hline$\hat{F}$ & empirical population randomly drawn from a population $F$ \\
\hline$F$ & population of observations \\
\hline$f$ & ellipsoid flattening \\
\hline$f(S)$ & arbitrary function of the DEM slope $S$ \\
\hline$H$ & orthometric height (m) \\
\hline$h$ & ellipsoidal height (m) \\
\hline$L$ & isometric latitude (rad) \\
\hline$\vec{n}$ & ellipsoid normal \\
\hline$N$ & length of the ellipsoid normal (m) \\
\hline$n$ & number of observations \\
\hline$P$ & horizontal plane \\
\hline$p$ & arbitrary DEM pixel \\
\hline$r$ & normally distributed random variable centered on 0 \\
\hline$R_{D E M}^{x}$ & DEM metric resolution along $x$ axis (m) \\
\hline$R_{D E M}^{y}$ & DEM metric resolution along $y$ axis (m) \\
\hline$S$ & slope of a DEM point \\
\hline$s_{\varepsilon}$ & standard deviation of the empirical population $\hat{E}$ \\
\hline$T_{L}$ & Linke turbidity factor \\
\hline$\alpha$ & angular elevation (rad) \\
\hline$\beta$ & strictly positive constant \\
\hline$\gamma$ & azimuth (rad) \\
\hline \multicolumn{2}{|r|}{$\Delta_{W G S 84-E G M 96}$ geoid undulation with respect to the ellipsoid (m) } \\
\hline$\Delta \lambda$ & longitude width of the DEM tile used in horizon computation (rad) \\
\hline$\Delta \varphi$ & latitude height of the DEM tile used in horizon computation (rad) \\
\hline $\bar{\varepsilon}$ & mean of the empirical population $\hat{E}$ \\
\hline$\varepsilon$ & horizon modeling error \\
\hline$\hat{\Theta}$ & estimator of a given population \\
\hline
\end{tabular}




\begin{tabular}{|ll|}
\hline$\hat{\theta}$ & parameter of a sample drawn from a given population \\
$\theta$ & parameter of a given population \\
$\lambda$ & geographic longitude (rad) \\
$\mu$ & mean of a given population \\
$v$ & significance level \\
$\rho$ & radius of curvature in the north-south direction $(\mathrm{m})$ \\
$\sigma$ & standard deviation of a given population \\
$\sigma^{2}$ & variance of a given population \\
$\varphi$ & geographic latitude (rad) \\
\hline
\end{tabular}

\section{Introduction}

At the present time, the international energy situation is composed of two related main issues: global warming and depletion of fossil resources (Ben Ahmed et al., 2011; Hegerl et al., 2007). Since the advent of the sustainable development concept at the end of the 1980s, many political decisions have been implemented around the world in order to reduce the environmental impact of human development (Liébard and De Herde, 2005). Thereby, the use of renewable resources, which emit very few Green House Gases (GHG) and are almost unlimited energies (Ben Ahmed et al., 2011), is one of the first objectives of the Kyoto Protocol established in 1997 (United Nations, 1998). Among many other kinds of renewable energies, direct solar energy is currently used through different conversion technologies mainly to produce heat or electricity (Arvizu et al., 2011), such as photovoltaics (Luque and Hegedus, 2003), flat solar thermal collectors (Mosallat et al., 2013), concentrated solar power (Viebahn et al., 2010), solar cooking (Wentzel and Pouris, 2007) or solar air conditioning (Bermejo et al., 2010).

In order to predict reliability of these systems for supplying energy to rural or urban populations, it is necessary to accurately assess both the significance and distribution of the resource, i.e. to map the solar potential. The consistent solar mapping from ground measurements requires the distance between measuring sensors to be very low (Muselli et al., 1998; Perez et al., 1997), which almost never happens (Paulescu et al., 2013). Therefore, many models have been developed over the past years in order to retrieve solar radiation reaching the ground from satellite observations, like the Heliosat model (Beyer et al., 1996; Rigollier, 2004), or the ones developed by Brisson et al. (1999), Zelenka et al. (1999), Perez et al. (2002) or Janjai et al. (2005). Nevertheless, if the accuracy of these models is now well established (Paulescu et al., 2013), all of them only estimate ground solar irradiance from the extraterrestrial radiation and a physical characterization of the atmospheric transmittance, and leave out, because of their inherent spatial resolution, interaction of the radiation with the local topography.

Some models, part of Geographic Information Systems (GIS) like ArcGIS or GRASS, already take into account local terrain effects in the computation of solar radiation (Ruiz-Arias et al., 2009). However, these models are mainly based on 
simplified atmospheric parameterizations (Ruiz-Arias et al., 2010, 2009), which still limits their consistency for developing regional solar map databases. As a result, in order to take benefit from the precision of the satellite-derived computation of the atmospheric transmittance, some post-processing procedures integrating topographic effects into satellite-based radiation maps have been proposed (Bosch et al., 2010; Haurant et al., 2012; Pillot et al., 2013; Ruiz-Arias et al., 2010). Finally, comparing estimates with ground data in mountainous areas, studies of Ruiz-Arias et al. (2010), Bosch et al. (2010) and Haurant et al. (2012) have shown this method improves geographic information and accuracy of the final solar radiation maps.

In order to evaluate terrain effects, this process requires the use of a numerical topographic map of the study region, i.e. a digital elevation model (DEM). First and main step of the procedure consists in retrieving the local horizon for every pixel of the DEM, in order to estimate diffuse and direct shading effects caused by the terrain onto the radiation (Dozier and Frew, 1990; Quaschning and Hanitsch, 1998). Thus, the higher the DEM resolution, the better the estimate accuracy, but also the longer the global computation, which is a very important parameter to take into consideration when the area of interest gets large, such as a small country (Pillot, 2014; Pillot et al., 2013). As a result, the authors think the corresponding horizon model has to present both accuracy and fast computing features, with in addition the possibility of evaluating model-based as well as DEM-based errors made on horizon estimates.

Consequently, we have developed a new efficient horizon numerical model using the Matlab programming language. In order to be fully implementable with exactly the same accuracy at any world location, it is exclusively based on theoretical assumptions without any empirical approximations. We have also added to this model a characteristic viewing distance computation allowing processing optimization, i.e. reduction of the model's running time by knowingly altering model precision. We have then compared resulting estimates with in situ data, collected during an original topographic measurement campaign, and with estimates from some other models in order to assess how relevant this new model was. At last, from the ground data comparison, we propose a first approximation method for predicting the DEM-based error achieved on horizon estimates.

This article is divided into 3 main parts: the first focuses on the characteristics of the DEM used in this work; the second depicts the model theoretical basis as well as the related horizon viewing distance and the proposed processing optimization method; the final part presents both the comparison and the DEM-based error prediction studies.

\section{DEM features}

Horizon modeling directly depends on which kind of DEM is employed and on which specific Earth's mathematical representation it is defined from. We therefore present here characteristics of the main DEM used at present time, retrieved from the global Shuttle Radar Topography Mission (SRTM), as well as the coordinate reference system in which elevation data are represented.

\subsection{The Shuttle Radar Topography Mission (SRTM): an interferometric measurement of the Earth's topography}

A lot of developed countries own large national cartographic databases which were used before 2000 to produce DEM. However, these DEM presented some limitations such as, on the one hand, country-specific resolution, scale and geographic projection, and, on the other hand, a low coverage of the global land because of some regions without any good-quality 


\footnotetext{
${ }^{1}$ At the time this study was driven, worldwide SRTM-1 DEM was not available yet. It has been meanwhile released and is now available from the NASA/USGS website (https://lta.cr.usgs.gov/SRTM1Arc).
}

topographic maps (Farr et al., 2007). Therefore, the National Aeronautics and Space Administration (NASA) in collaboration with the National Geospatial-Intelligence Agency (NGA) prepared and achieved the SRTM, in order to propose a new global and consistent high-quality representation of the Earth's topographic surface.

The SRTM lasted 10 days in February 2000 and consisted in radar scanning, from the space shuttle Endeavour, of $80 \%$ of the world emerged lands located between $60^{\circ} \mathrm{N}$ and $57^{\circ} \mathrm{S}$ (Farr et al., 2007; Rabus et al., 2003). The use of an interferometric synthetic aperture radar (InSAR), operating in the $\mathrm{C}$ band (wavelength $=5.6 \mathrm{~cm}$ ), has permitted the assessment of land topography by measuring phase difference between radar pulses reflected by the surface and received at the same time by 2 distinct antennas (Farr et al., 2007; Jarvis et al., 2004). Earth's DEM surface computed from SRTM data is a regular altitude mesh at 1" resolution (SRTM-1 DEM, about $30 \mathrm{~m}$ near the equator), presenting an absolute vertical error lower than $9 \mathrm{~m}$ (90 \% confidence interval).

First SRTM datasets were released in 2003 by the NASA, with the SRTM-1 DEM mesh available only for the United States $^{1}$. Indeed, the rest of the world is covered by the DEM at $3^{\prime \prime}$ resolution (SRTM-3 DEM, about $90 \mathrm{~m}$ near the equator), computed by averaging $3 \times 3$ pixels of the original SRTM-1 DEM product. Furthermore, a significant proportion of elevation data is still missing in the original DEM, corresponding to a total area of $796217 \mathrm{~km}^{2}$ (Jarvis et al., 2008) and mainly resulting from the low contrast within the final image (sharp slopes) or from smooth areas (smooth water or sand) which reflect too little energy to the radar (Farr et al., 2007). Several methods have been developed to fill in these voids, mainly based on interpolation algorithms and on the use of elevation data from other sources. These schemes have been integrated into a global diffusion tool developed by the Consortium for Spatial Information (CGIAR-CSI), available via the organization website (Jarvis et al., 2008). This tool gives access to SRTM-3 DEM $5^{\circ} \times 5^{\circ}$ tiles we have used in this work.

\subsection{SRTM DEM coordinate reference systems}

A DEM is a numerical mesh of the Earth's topographic surface where geographic coordinates of each pixel depend on the mathematical shape used to represent it. The Earth's shape is not a perfect sphere because of the flattening at the poles and the heterogeneous gravity field at the surface. Therefore, 2 coordinate systems have been used by the SRTM to produce and reference elevation data: the WGS84 ellipsoid of revolution for horizontal position (geographic, or geodetic, longitude and latitude) and the EGM96 geoid for vertical elevation (orthometric height).

\subsubsection{Geographic coordinates: the WGS84 ellipsoid}

The World Geodetic System 1984 (WGS84) is a geocentric, right-handed and orthogonal Earth coordinate system, where origin $O$ is the globe's center of mass, $z$ axis is the axis passing through the poles and $x$ axis is the intersection between the reference meridian (Greenwich) and the plane normal to the $z$ axis and passing through $O$ (NGA, 2000). The WGS84 ellipsoid of center $O$ is a mathematical approximation of the Earth's shape retrieved by revolution, around its semi-minor axis $b$, of an ellipse of semi-major axis $a$ and flattening $f=\frac{a-b}{a}$ : 


$$
\begin{aligned}
& a=6378137.0 \mathrm{~m} \\
& f=1 / 298.257223563
\end{aligned}
$$

The ellipsoid is fully defined by these 2 parameters, from which we can also express the first eccentricity $e$ (Bosser, 2012; Burkholder, 2008):

$$
e^{2}=\frac{a^{2}-b^{2}}{a^{2}}=2 f-f^{2}
$$

Let now $M$ be a point of the Cartesian coordinate system $(x, y, z)$ and $M^{*}$ the orthogonal projection of $M$ onto the ellipsoid such as $\overrightarrow{M^{*} M}=h \cdot \vec{n}$, with $\vec{n}$ the normal to the ellipsoid in $M^{*}$ (Bosser, 2012). In the geographic coordinate system, $M$ is defined by its latitude $\varphi$, angle between $\vec{n}$ and the equatorial plane, its longitude $\lambda$, angle between the reference meridian and the meridian passing through $M^{*}$, and its ellipsoidal height $h$ along the normal. In order to retrieve the coordinates of $M$ in the Cartesian space from the geographic space, it is firstly possible to express the normal to the ellipsoid as in the case of a sphere:

$$
\vec{n}=\left(\begin{array}{c}
\cos \lambda \cos \varphi \\
\sin \lambda \cos \varphi \\
\sin \varphi
\end{array}\right)
$$

Then, we can calculate the Cartesian coordinates $x, y, z$ of any point $M$ onto the ellipsoid from its geographic coordinates $\varphi, \lambda, h$ (Bosser, 2012; Burkholder, 2008):

$$
\left\{\begin{array}{l}
x=(N+h) \cos \lambda \cos \varphi \\
y=(N+h) \sin \lambda \cos \varphi \\
z=\left(N\left(1-e^{2}\right)+h\right) \sin \varphi
\end{array}\right.
$$

Where $N$ is the length of the ellipsoid normal, i.e. the distance between $z$ axis and $M^{*}$ along the ellipsoid normal $\vec{n}$ (Burkholder, 2008):

$$
N=\frac{a}{\sqrt{1-e^{2} \sin ^{2} \varphi}}
$$

Figure 1 shows the WGS84 ellipsoid in the Cartesian coordinate system. $A\left(\varphi_{A}, \lambda_{A}, h_{A}\right)$ and $B\left(\varphi_{B}, \lambda_{B}, h_{B}\right)$, with corresponding distances $N_{A}$ and $N_{B}$ from $z$ axis, are 2 points of the Cartesian space such as $\overrightarrow{A^{*} A}=h_{A} \cdot \overrightarrow{n_{A}}$ and $\overrightarrow{B^{*} B}=h_{B} \cdot \overrightarrow{n_{B}}$, and will be used later to explain horizon modeling.

\subsubsection{Altitude baseline: the EGM96 geoid}

A geoid is a mathematical model defining a specific equipotential surface of the Earth's gravity field around the mean sea level (NGA, 2000), and is used as a baseline to retrieve altitude of the topographic surface. The Earth Gravitational Model 


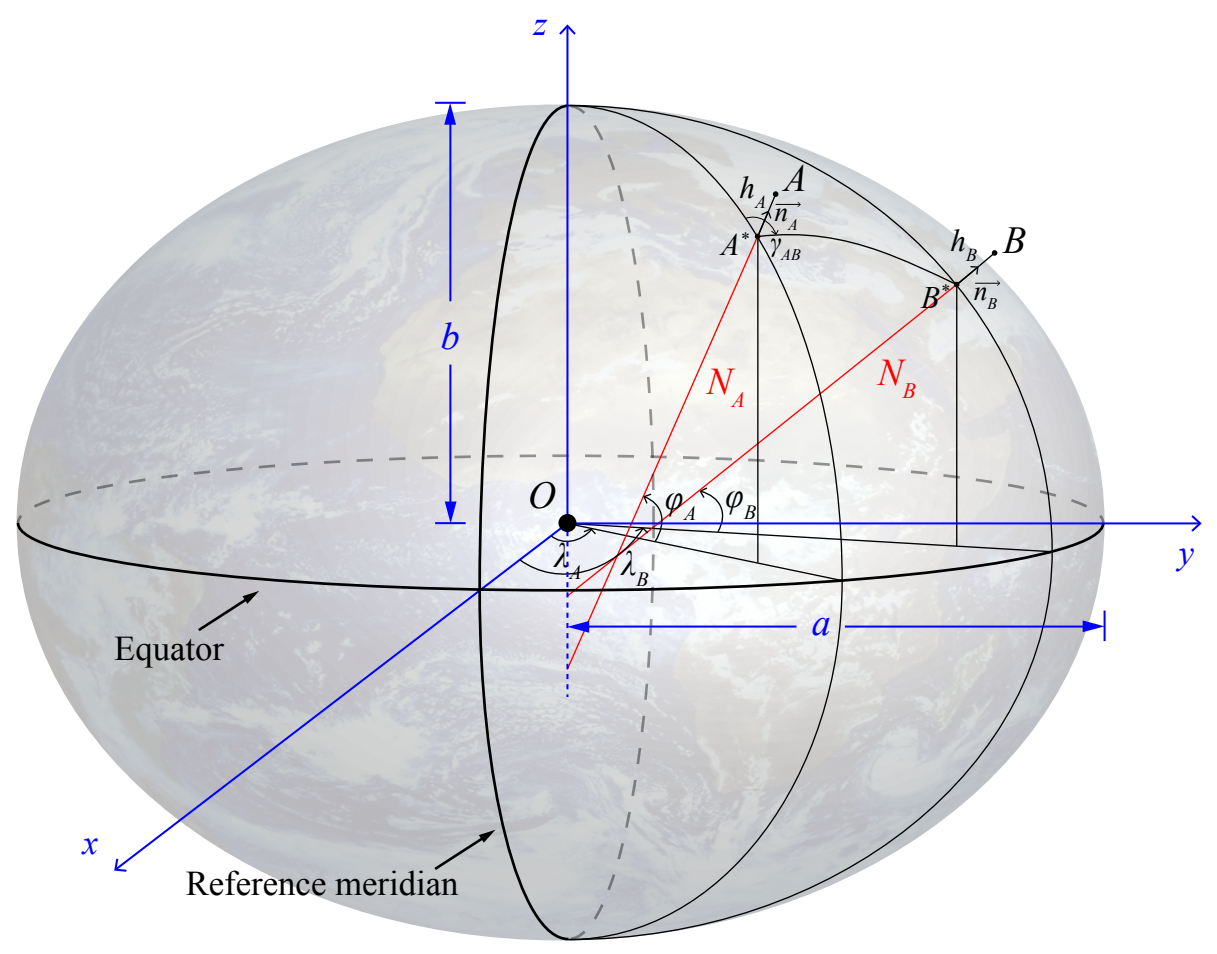

Figure 1: WGS84 ellipsoid of revolution used as the reference geographic coordinate system for the SRTM DEM.

1996 (EGM96) has been developed by the NASA and NGA in order to evaluate spatial variations of the gravity field, i.e. to determine surfaces where it remains constant (Lemoine et al., 1998). Undulations of this geoid referring to the WGS84 ellipsoid, i.e. the distance between both the mathematical shapes in any point, can be determined using the model provided by the NGA (NGA, 2014). It is thus possible, for any point of the DEM, to derive the geoid-based altitude or orthometric height $H$ from the ellipsoidal height $h$ considering the geoid undulation $\Delta_{W G S 84-E G M 96}$ (Lemoine et al., 1998; NGA, 2000):

$$
h=H+\Delta_{W G S 84-E G M 96}
$$

The gap $\Delta_{W G S 84-E G M 96}$ is positive or negative according to the geoid position above or under the ellipsoid, and remains between approximately $-107 \mathrm{~m}$ and $85 \mathrm{~m}$ (NGA, 2000).

\section{Horizon modeling}

Obstruction induced by the Earth's topography modifies the local horizon and thus the solar radiation reaching the ground. It is defined, in horizontal coordinates, by the azimuth $\gamma$ and the angular elevation $\alpha$. Figure 2 depicts the horizon observed from $A\left(\varphi_{A}, \lambda_{A}, h_{A}\right)$ and including $B\left(\varphi_{B}, \lambda_{B}, h_{B}\right)$, defined in the horizontal plane $P$ with respect to $A$ by its azimuth $\gamma_{A B}$ and its elevation $\alpha_{A B}$. DEM-based horizon modeling consists therefore in computing elevation $\alpha$ and azimuth $\gamma$ of all the points of the DEM around the observation point, and then retrieving the maximum $\alpha$ for every azimuth between 0 and $360^{\circ}$. 


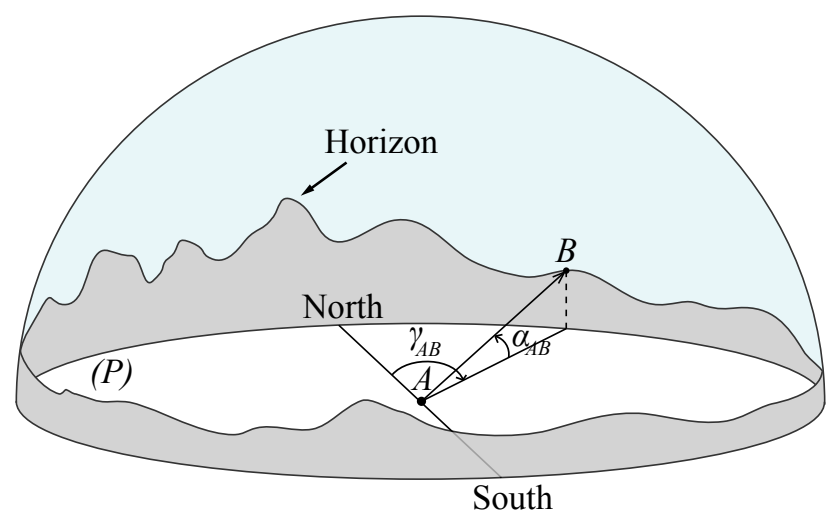

Figure 2: Obstructed horizon, induced by the terrain topography, represented in the horizontal coordinates $(\gamma, \alpha)$ of the plane $P$ centered on $A$.

\subsection{Horizon theoretical model}

Here we present the horizon model we have developed considering both the points $A$ and $B$ already described in Figure 1 and in Figure 2.

\subsubsection{Ellipsoidal height}

Our theoretical model is based on both the reference systems previously described (WGS84 and EGM96). Every point of a SRTM DEM is thus defined by its geographic coordinates and its orthometric height (Farr et al., 2007). Nevertheless, if the angular elevation $\alpha_{A B}$ depends on the height $H_{B}$ as well as on the height $H_{A}$, they are not both computed with respect to the same baseline because of the variations of the gravity field near the Earth's surface (geoid). Therefore, in order to accurately evaluate $\alpha_{A B}$ from the DEM, it is necessary to retrieve altitude of the 2 points with respect to a same regular surface such as the WGS84 ellipsoid. As described before, it is possible to derive ellipsoidal heights $h_{A}$ and $h_{B}$ from relation (6):

$$
\begin{aligned}
& h_{A}=H_{A}+\Delta_{W G S 84-E G M 96}(A) \\
& h_{B}=H_{B}+\Delta_{W G S 84-E G M 96}(B)
\end{aligned}
$$

$\Delta_{W G S 84-E G M 96}(A)$ and $\Delta_{W G S 84-E G M 96}(B)$ are geoid undulations with respect to the ellipsoid in $A$ and $B$ respectively, and are depicted in Figure 3.

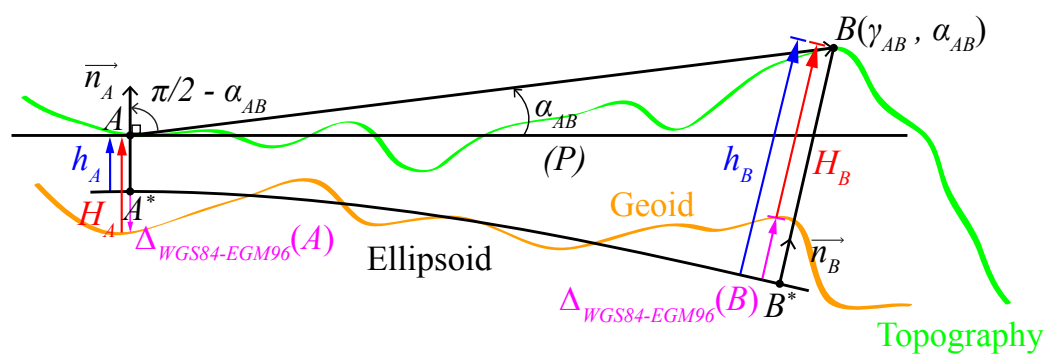

Figure 3: Sectional view of the ellipsoid surface in the $A B$ direction. 


\subsubsection{Angular elevation}

Figure 3 is a sectional view of the ellipsoidal surface along the direction $A B$ where belong the geoid, the terrain topography and the horizontal plane $P$ tangent to the ellipsoid in $A$ (orthogonal to $\overrightarrow{n_{A}}$ ). The fastest method to calculate the angular elevation $\alpha_{A B}$, i.e. the angle between the vector $\overrightarrow{A B}$ and the plane $P$, is probably to use the scalar product. Indeed, $\alpha_{A B}$ can be computed coupling the scalar product between the normal $\overrightarrow{n_{A}}$ and the vector $\overrightarrow{A B}$ to the angle between them:

$$
\overrightarrow{n_{A}} \cdot \overrightarrow{A B}=\left\|\overrightarrow{n_{A}}\right\| \cdot\|\overrightarrow{A B}\| \cdot \cos \left(\frac{\pi}{2}-\alpha_{A B}\right)
$$

Obstruction only exists if the angular elevation is positive; so, using the sine, we finally have:

$$
\alpha_{A B}=\max \left(\arcsin \left(\frac{\overrightarrow{n_{A}} \cdot \overrightarrow{A B}}{\|\overrightarrow{A B}\|}\right), 0\right)
$$

In the Cartesian coordinate system, $\overrightarrow{n_{A}}$ is derived from the relation (3). Then, equation (4) computes the coordinates $\left(x_{A}, y_{A}, z_{A}\right)$ and $\left(x_{B}, y_{B}, z_{B}\right)$ of $A$ and $B$, which can be used to express the vector $\overrightarrow{A B}$ and its norm:

$$
\overrightarrow{A B}=\left(\begin{array}{c}
x_{B}-x_{A} \\
y_{B}-y_{A} \\
z_{B}-z_{A}
\end{array}\right) \text { and }\|\overrightarrow{A B}\|=\sqrt{\left(x_{B}-x_{A}\right)^{2}+\left(y_{B}-y_{A}\right)^{2}+\left(z_{B}-z_{A}\right)^{2}}
$$

\subsubsection{Azimuth}

In horizontal coordinates, azimuth $\gamma$ is the rotation angle between the reference north direction and the direction of interest. Azimuth $\gamma_{A B}$ of the point $B$ with respect to the observation point $A$ is represented on the ellipsoid of Figure 1 as well as on the horizontal plane of Figure 2. It is possible to retrieve the azimuth $\gamma$ using the meridian and parallel line elements $d s_{m}$ and $d s_{p}$ depicted in Figure 4, such as (Bosser, 2012):

$$
\tan \gamma=\frac{d s_{p}}{d s_{m}}
$$

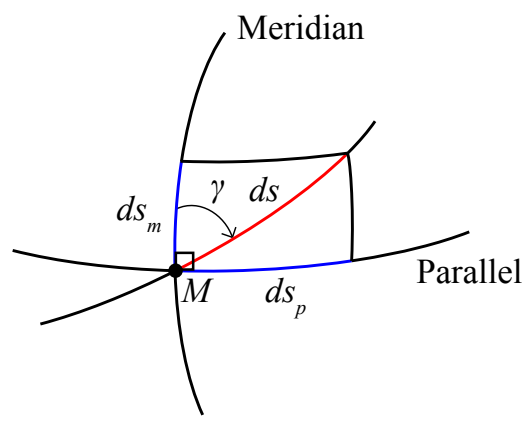

Figure 4: Azimuth $\gamma$ of a line drawn on the ellipsoid and calculated from meridian $\left(d s_{m}\right)$ and parallel $\left(d s_{p}\right)$ line elements (Bosser, 2012). 
Each line element depends on the considered geographic coordinate system $(\lambda, \varphi)$, which is not a symmetric parameterization, i.e. the same angular variation $(d \lambda=d \varphi)$ doesn't lead to the same travel onto the surface $\left(d s_{p} \neq d s_{m}\right)$. A symmetric parameterization $(\lambda, L)$ of the ellipsoid is thus defined as follows:

$$
\frac{d s_{p}}{d s_{m}}=\frac{d \lambda}{d L}
$$

Where $L$ is the isometric latitude, undefined at the poles $(\varphi= \pm \pi / 2)$, and which is retrieved from the geographic latitude $\varphi$ by applying the first fundamental quadratic form to the ellipsoid of revolution (Bosser, 2012; Eisenhart, 1909):

$$
L=\ln \left[\tan \left(\frac{\pi}{4}+\frac{\varphi}{2}\right) \cdot\left(\frac{1-e \sin \varphi}{1+e \sin \varphi}\right)^{\frac{e}{2}}\right]
$$

So, finally, the azimuth $\gamma_{A B}$ is determined by integrating equation (12) along the loxodrome $A B$, i.e. the constant azimuth line between $A$ and $B$ :

$$
\gamma_{A B}=\arctan \left(\frac{\lambda_{B}-\lambda_{A}}{L_{B}-L_{A}}\right)
$$

\subsubsection{Computing full horizon}

We have just defined the angular elevation $\alpha_{A B}$ and the azimuth $\gamma_{A B}$ of a DEM point $B$ with respect to an observation point $A$. In order to compute the full horizon, it is therefore necessary to apply the process to all the terrain which can be viewed from this point. Once it is perfectly characterized in the horizontal coordinate system $(\gamma, \alpha)$, it is then possible to identify, for a complete $360^{\circ}$ azimuth rotation, every maximal angular elevation, and so to retrieve the horizon.

From relations (9) and (14), a DEM originally referenced in the geographic coordinate system $(\lambda, \varphi, h)$ can be now represented in the new horizontal coordinate system $(\gamma, \alpha)$. Furthermore, InSAR technology collects surface altitude: the SRTM DEM is therefore an elevation regular mesh at $1^{\prime \prime}$ or $3^{\prime \prime}$ resolution, composed of square pixels referenced by the geographic coordinates of their geometric center (Farr et al., 2007; Jarvis et al., 2004).

As shown in Figure 5, the first step of the process consists in demarcating a new narrower DEM tile, characterized by longitude width $\Delta \lambda$ and latitude height $\Delta \varphi$, around a given observation point $A\left(\lambda_{A}, \varphi_{A}, h_{A}\right)$. The size of this new area will determine the horizon computation time and will be defined in the next section. Once the local terrain mesh is perfectly known, the angular elevation $\alpha$ of each pixel is retrieved applying equation (9) to the geographic coordinates of its center $M(\lambda, \varphi, h)$.

As described in Figure 5, a loxodrome $\gamma_{A F}$ drawn from $A$ to any boundary point $F$ passes through a finite number of pixels $\left[p_{1}, p_{2}, \ldots, p_{i}\right]$ with angular elevation $\left[\alpha_{1}, \alpha_{2} \ldots, \alpha_{i}\right]$. Along this line, the point $B$ presents the highest elevation $\alpha_{B}$ and thus belongs to the horizon observed from $A$. To detect the pixels belonging to the loxodrome, our model calculates for each pixel of center $M(\gamma, \alpha)$ azimuth of northwest, southwest and southeast corners $\gamma_{N W}, \gamma_{S W}$ and $\gamma_{S E}$ using relation (14). Then, the azimuth line passes through the pixel if $\gamma_{A F}$ is between $\gamma_{N W}$ and $\gamma_{S W}$ along a meridian, and between $\gamma_{S W}$ and $\gamma_{S E}$ along a parallel. Once elevation of all the pixels of the line is computed, the maximum is easily retrieved and corresponds to the horizon elevation in the $\gamma_{A F}$ azimuth direction. 


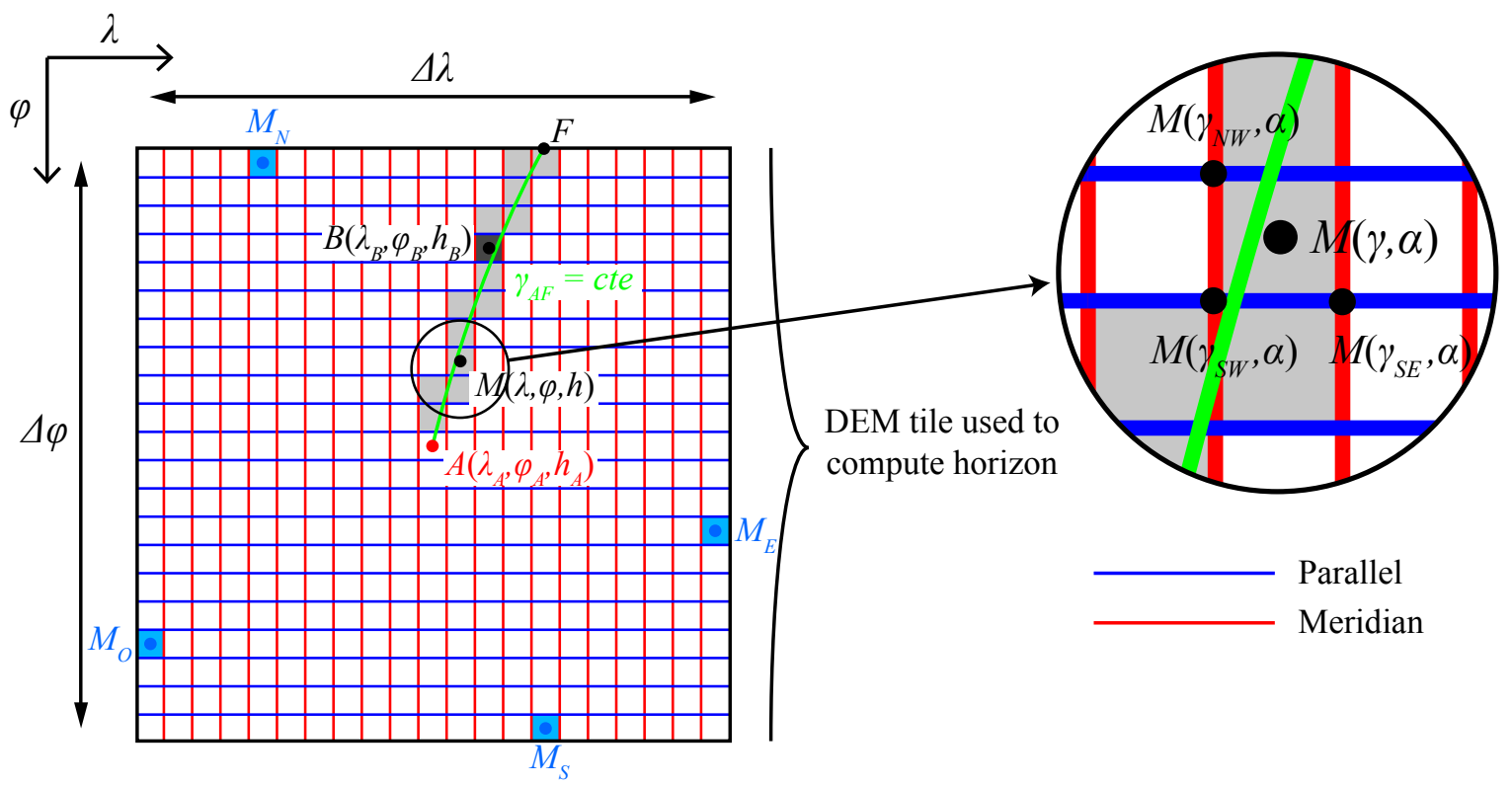

Figure 5: Methodology for computing the horizon around a given point $A$. Within a specific DEM tile of size $(\Delta \lambda, \Delta \varphi)$ centered on $A$, and for each azimuth between $0^{\circ}$ and $360^{\circ}$, it consists in retrieving the pixel with the highest elevation (here $\alpha_{B}$ ) among all the pixels the given loxodrome passes through (here $\left.\gamma_{A F}\right)$.

Finally, computing the full horizon consists in repeating this step for a set of azimuth lines $\left[\gamma_{A F_{1}}, \gamma_{A F_{2}}, \ldots, \gamma_{A F_{i}}\right]$. Obviously, the selected number of lines directly influences horizon accuracy and computing time: in this study, we have considered a horizon azimuth step of $1^{\circ}$.

\subsection{Characteristic horizon viewing distance and model optimization}

We present here an innovative way to reduce the size of the DEM tile required for accurately computing a single horizon, as well as a related method allowing us to save computation time in the case of large geographic areas, by knowingly altering the precision of multiple estimated horizons.

\subsubsection{Characteristic viewing distance}

Most horizon numerical models, whether specialized as the Carnaval French software or included in a GIS as the r.horizon model (Hofierka et al., 2007) part of the r.sun add-on of the GRASS GIS software (Šúri and Hofierka, 2004), let the user define the maximal distance used to compute the horizon around the observation point. However, the Earth's roundness inherently limits the minimum altitude of any DEM point: beyond a given distance from the observation point, this point is then necessarily located below the horizontal plane $P$. We can observe this statement in Figure 3 where, beyond a specific angle between $\overrightarrow{n_{A}}$ and $\overrightarrow{n_{B}}$, the angular elevation $\alpha_{A B}$ turns negative.

Consequently, we have developed a characteristic viewing distance concept, i.e. the maximum distance beyond which any DEM elevation data is no longer useful to compute a given horizon with the highest possible accuracy. In our model, the DEM 
tile used to model the horizon has width $\Delta \lambda$ and height $\Delta \varphi$, and is centered on an observation point defined in the geographic coordinate system $(\lambda, \varphi)$. The aim was therefore to define a characteristic viewing area for the horizon computation, i.e. the largest efficient DEM tile of width $\Delta \lambda_{\max }$ and height $\Delta \varphi_{\max }$ beyond which angular elevation of any DEM pixel is necessarily negative or 0 .

To do this, it is firstly possible to express relations allowing calculation of the angle $\varphi-\varphi_{A}$ along a meridian (constant longitude), and the angle $\lambda-\lambda_{A}$ along a parallel (constant latitude), between any point $M(\lambda, \varphi, h)$ of normal length $N$ and the observation point $A\left(\lambda_{A}, \varphi_{A}, h_{A}\right)$ of normal length $N_{A}$, where $h>h_{A}$, such as $\alpha_{A M}=0$. The angular elevation being equal to 0 when the normal $\overrightarrow{n_{A}}$ is perpendicular to the vector $\overrightarrow{A M}$, it leads to the 2 following equations:

$$
\begin{aligned}
& \left(\overrightarrow{n_{A}} \cdot \overrightarrow{A M}\right)_{\lambda=\lambda_{A}}=0 \\
& \left(\overrightarrow{n_{A}} \cdot \overrightarrow{A M}\right)_{\varphi=\varphi_{A}}=0
\end{aligned}
$$

From the revolution symmetry of the ellipsoid results an explicit solution to the equation (16):

$$
\left|\lambda-\lambda_{A}\right|_{\varphi=\varphi_{A}}=\left.\delta \lambda(h)\right|_{\varphi}=\arccos \left(\frac{\left(N_{A}+h_{A}\right) \cos ^{2} \varphi_{A}-\left(h-h_{A}\right) \sin ^{2} \varphi_{A}}{\left(N_{A}+h\right) \cos ^{2} \varphi_{A}}\right)
$$

Meanwhile, asymmetry of the meridian ellipse leads to an implicit formula for the angle $\varphi-\varphi_{A}$ :

$$
(N+h) \cos \left(\varphi-\varphi_{A}\right)+\left(N_{A} \sin \varphi_{A}-N \sin \varphi\right) e^{2} \sin \varphi_{A}-\left(N_{A}+h_{A}\right)=0
$$

While this equation can be numerically solved (iteration, dichotomy), it is however more interesting to use a local approximation in order to obtain an explicit solution. This is done by regarding the meridian ellipse as a circle where the radius is the radius of curvature in the north-south direction (Bosser, 2012; Burkholder, 2008). The middle term of the previous equation disappears (eccentricity equal to 0 ) and the lengths of the ellipsoid normal $N_{A}$ and $N$ are replaced by the radius $\rho$ of the local spherical approximation calculated in $A$ :

$$
\left|\varphi-\varphi_{A}\right|_{\lambda=\lambda_{A}}=\left.\delta \varphi(h)\right|_{\lambda}=\arccos \left(\frac{\rho+h_{A}}{\rho+h}\right)
$$

Where $\rho$ is given by the following relation (Bosser, 2012; Burkholder, 2008):

$$
\rho=\frac{a\left(1-e^{2}\right)}{\left(1-e^{2} \sin ^{2}\left(\varphi_{A}\right)\right)^{\frac{3}{2}}}
$$

In that specific case, $M$ can be any point located whether on the meridian or on the parallel passing through $A$. But we can easily generalize these 2 relations to any boundary point of coordinates $\left(\lambda_{A} \pm\left.\delta \lambda(h)\right|_{\varphi}, \varphi_{A} \pm\left.\delta \varphi(h)\right|_{\lambda}, h\right)$ since we will still have $\alpha_{A M} \leq 0$. It is then possible to use this methodology to retrieve the size $\left(\Delta \lambda_{\max }, \Delta \varphi_{\max }\right)$ of the largest efficient DEM tile used to build the horizon. Instances of boundary points in the 4 cardinal directions, $M_{N}\left(\lambda_{M_{N}}, \varphi_{M_{N}}, h_{M_{N}}\right)$ to the north, 
$M_{E}\left(\lambda_{M_{E}}, \varphi_{M_{E}}, h_{M_{E}}\right)$ to the east, $M_{S}\left(\lambda_{M_{S}}, \varphi_{M_{S}}, h_{M_{S}}\right)$ to the south and $M_{W}\left(\lambda_{M_{W}}, \varphi_{M_{W}}, h_{M_{W}}\right)$ to the west, are depicted in Figure 5 and are retrieved by iteration. To do this, we firstly initiate heights $h_{M_{N}}, h_{M_{E}}, h_{M_{S}}$ and $h_{M_{W}}$ with the maximum theoretical value of the global DEM (Everest's altitude for instance). Then, we iteratively reduce the size of the optimal DEM tile, by looking for the maximum height in each cardinal direction within the remaining area, as long as one of the following conditions is still met:

$$
\left\{\begin{array}{lll}
0 \leqslant \varphi_{M_{N}}-\varphi_{A} \leqslant\left.\delta \varphi\left(h_{M_{N}}\right)\right|_{\lambda} & \& & \lambda_{M_{W}} \leqslant \lambda_{M_{N}} \leqslant \lambda_{M_{E}} \\
0 \leqslant \varphi_{A}-\varphi_{M_{S}} \leqslant\left.\delta \varphi\left(h_{M_{S}}\right)\right|_{\lambda} & \& & \lambda_{M_{W}} \leqslant \lambda_{M_{S}} \leqslant \lambda_{M_{E}} \\
0 \leqslant \lambda_{A}-\lambda_{M_{W}} \leqslant\left.\delta \lambda\left(h_{M_{W}}\right)\right|_{\varphi} & \& & \varphi_{M_{S}} \leqslant \varphi_{M_{W}} \leqslant \varphi_{M_{N}} \\
0 \leqslant \lambda_{M_{E}}-\lambda_{A} \leqslant\left.\delta \lambda\left(h_{M_{E}}\right)\right|_{\varphi} & \& & \varphi_{M_{S}} \leqslant \varphi_{M_{E}} \leqslant \varphi_{M_{N}}
\end{array}\right.
$$

Finally, the width and height of the optimal DEM tile required to perfectly characterize the horizon are given by:

$$
\begin{aligned}
& \Delta \varphi_{\max }=\varphi_{M_{N}}-\varphi_{M_{S}} \\
& \Delta \lambda_{\max }=\lambda_{M_{E}}-\lambda_{M_{W}}
\end{aligned}
$$

Obviously, in mapping applications, it is not really efficient to define a characteristic area for every single computed horizon of a given region. But it is possible to define the optimal DEM of a specific region, i.e. the zone beyond which it is no longer necessary to look for elevation data to compute any horizon of the region with the highest possible accuracy. In that case, we no longer consider a single point but a geographic rectangle enclosing the boundaries of the study region; the optimal DEM tile is then retrieved by using the previous method and by regarding $h_{A}$ as the minimum height inside the region. This tile encompasses the region enough for allowing the computation of all horizons with the highest possible accuracy.

\subsubsection{Reducing model's running time}

Depending on the size of the considered region, the desired accuracy and the purpose of the study, it may be interesting to reduce the model's computation time in order to improve efficiency of the whole process. Moreover, now both the optimal DEM and the maximum characteristic area of a given region are defined, it is possible to knowingly decrease accuracy of a given horizon by comparing it with the theoretical ideal one. We therefore propose an efficient method allowing, in the case of large geographic areas, computation time to be reduced and resulting horizon precision to be estimated.

In order to avoid an optimization as time-consuming as the area processing itself, it is relevant to use statistical inferences, which consist in assessing the most significant properties of the global error distribution through a given territory by analyzing smaller data sets sampled from it.

Regarding statistical inferences, estimating the parameter $\theta$ of a population of observations $F$ consists in determining the estimator $\hat{\Theta}$, random variable equal to $\hat{\theta}$ for each sample we randomly pull out from $F$ (Walpole et al., 2011). However, in order to accurately assess the standard error and the confidence intervals of the sampling distribution of $\hat{\Theta}$, the distribution 
of the population $F$ also requires to be perfectly known (Walpole et al., 2011). Here, it was therefore necessary to look for a non-parametric method, i.e. fully independent of the distribution, remaining accurate in spite of the low sample size.

A well-suited method to that kind of problem is the bootstrap method (Efron and Tibshirani, 1993), which relies on the plug-in statistical principle. This principle consists in determining the parameter $\theta$ of any distribution $F$, such as $\theta=t(F)$, from the estimate $\hat{\theta}$ of an empirical distribution $\hat{F}$, which is a sample composed of $n$ observations randomly drawn from $F$, such as $\hat{\theta}=t(\hat{F})$. In addition, it is also necessary to apprehend the corresponding error, and here comes the main idea of the bootstrap concept. It consists in re-sampling $\hat{F}$, i.e. in drawing with replacement $B n$-sized random samples $\hat{F}^{*(b)}$ in $F$, with $b=1,2, \ldots, B$, called bootstrap samples. Then, the distribution of the $B$ boostrap sample estimates $\hat{\theta}^{*(1)}, \hat{\theta}^{*(2)}, \ldots, \hat{\theta}^{*(B)}$ around $\hat{\theta}$, such as $\hat{\theta}^{*(b)}=t\left(\hat{F}^{*(b)}\right)$, is used to calculate the standard error and the confidence interval of the estimate $\hat{\theta}$ (Efron and Tibshirani, 1993; Singh and Xie, 2010).

Let $\varepsilon$ be the error between an horizon estimate retrieved for a given viewing area $(\Delta \varphi, \Delta \lambda)$ and the ideal theoretical horizon corresponding to the optimal area $\left(\Delta \varphi_{\max }, \Delta \lambda_{\max }\right)$. Let then $E_{k}$ be the population of all the errors achieved on the calculation of each $k$-horizon, i.e. computed using a specific $k$-area of size $\left(\Delta \varphi_{k}, \Delta \lambda_{k}\right)$, within a given region. In order to determine the main components of the resulting error, i.e. both the mean $\mu_{E_{k}}$ and the variance $\sigma_{E_{k}}^{2}$ of the population $E_{k}$, a plug-in estimate of $n$ independent errors $\hat{E}_{k}=\left\{\varepsilon_{1}, \varepsilon_{2}, \ldots, \varepsilon_{n}\right\}_{k}$ is gathered by computing the errors of $n k$-horizons corresponding to $n$ random DEM pixels.

First of all, in order to define the horizon viewing area of a given region of interest, it is relevant to consider a low number of observations $(n \leq 50)$ and to repeat the previous step for different $k$-areas. Then, by using a box plot representing the error against the computation time, it is possible to select the best compromise between them. As an example, Figure 6 shows the box plot we have used to determine the most relevant area size for computing all horizons of the Republic of Djibouti (Pillot, 2014).

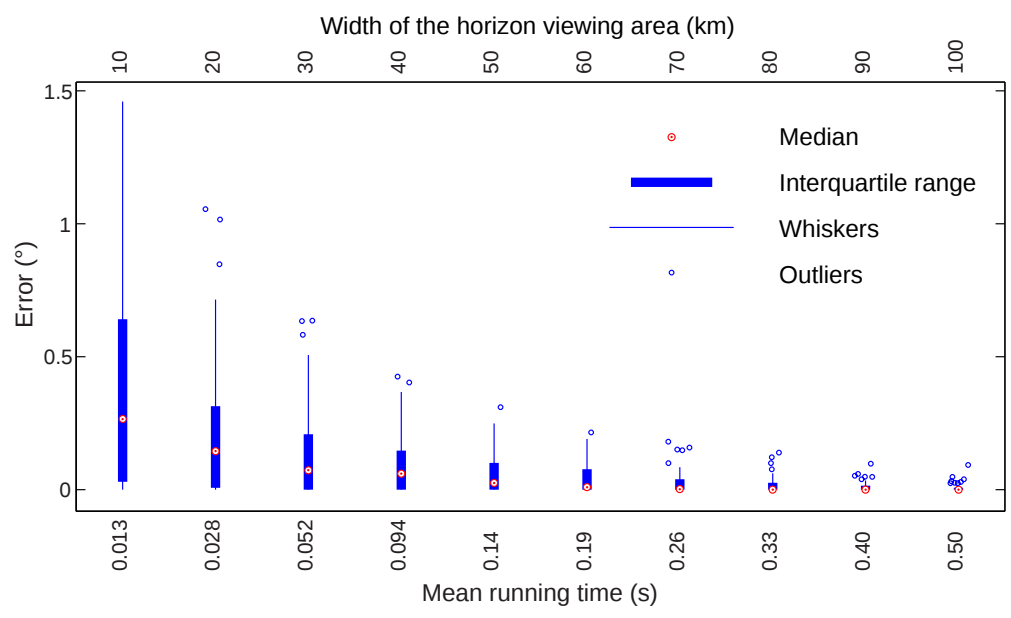

Figure 6: Example of the box plot used to optimize horizon calculation in the Republic of Djibouti, where model's error is plotted against mean running time for different k-areas (Pillot, 2014). In that example, the error determined with respect to the ideal horizon is the root mean square error. 
Once the desired $k$-area is known, it is then possible to evaluate the likely error performed on any corresponding horizon computed within the given region. In other words, it means estimating the mean $\mu_{E}$ and the standard deviation $\sigma_{E}$ of the distribution $E$ of the modeling error $\varepsilon$, which follows a probability law $\varepsilon \sim\left(\mu_{E}, \sigma_{E}^{2}\right)$. To perform this, a new empirical random sample $\hat{E}=\left\{\varepsilon_{1}, \varepsilon_{2}, \ldots, \varepsilon_{n}\right\}$ composed of a larger set of observations (for instance $n=200$ ) is implemented. From this empirical population, $B=n^{2}$ bootstrap samples $\hat{E}^{*(b)}=\left\{\varepsilon_{1}^{*}, \varepsilon_{2}^{*}, \ldots, \varepsilon_{n}^{*}\right\}$, where $b=1,2, \ldots, B$, are drawn with replacement (Singh and Xie, 2010). Thereafter, both the mean $\bar{\varepsilon}^{*(b)}$ and the standard deviation $s_{\varepsilon}^{*(b)}$ of each sample are calculated. Their distribution allows assessing the accuracy of the mean $\bar{\varepsilon}$ and the standard deviation $s_{\varepsilon}$ of the empirical population, which infer the parameters $\mu_{E}$ and $\sigma_{E}$ of the global population.

Finally, a simple method for estimating confidence intervals of the population parameters $\left(\mu_{E}\right.$ and $\left.\sigma_{E}\right)$ is the percentile method. However, asymmetry and bias of the standard deviation distribution (Walpole et al., 2011) make an approach such as the bias-corrected and accelerated $\left(\mathrm{BC}_{\mathrm{a}}\right)$ procedure, developed by (Efron, 1987), better suited. The classical percentile approach gives, for a significance level $v$, the $(1-2 v) \%$ confidence interval of the given estimation $\hat{\theta}$ as $\left[\hat{\theta}^{*(v)}, \hat{\theta}^{*(1-v)}\right]$, where $\hat{\theta}^{*(v)}$ is the $100 v^{\text {th }}$ percentile of the distribution of the $B$ bootstrap estimates $\hat{\theta}^{*(1)}, \hat{\theta}^{*(2)}, \ldots, \hat{\theta}^{*(B)}$. In order to correct both the bias and the asymmetry of the given estimator, the $\mathrm{BC}_{\mathrm{a}}$ method enhances this first approximation by using other interval boundaries, as $\left[\hat{\theta}^{*\left(v_{1}\right)}, \hat{\theta}^{*\left(v_{2}\right)}\right]$ where $v_{1}$ and $v_{2}$ are adjusted with respect to $v$ and $1-v$ using the standard normal cumulative distribution function (Efron, 1987; Efron and Tibshirani, 1993).

Accordingly, the resulting distribution $E$ of the horizon modeling error $\varepsilon$ will follow a probability law $\varepsilon \sim\left(\mu_{E}, \sigma_{E}^{2}\right)$ such as:

$$
\left\{\begin{array}{l}
\mu_{E} \in\left[\bar{\varepsilon}^{*\left(v_{1}\right)}, \bar{\varepsilon}^{*\left(v_{2}\right)}\right] \\
\sigma_{E} \in\left[s_{\varepsilon}^{*\left(v_{1}\right)}, s_{\varepsilon}^{*\left(v_{2}\right)}\right]
\end{array}\right.
$$

\section{Model's efficiency and DEM-based error prediction}

We have presented the theoretical basis of our horizon model and proposed a method to choose the best compromise between the computation time and the corresponding model's accuracy. Nevertheless, this precision is only related to the ideal DEM-based horizon and not to the real horizon, so the error induced by the not fully accurate DEM representation of the Earth's topography has not been considered yet. As a result, we assess here the global accuracy of the final horizon estimates and compare it to other existing models in order to evaluate our model's performances. Finally, we also propose a method for predicting the horizon's DEM-based error.

\subsection{Model's efficiency: accuracy vs computation time}

The efficiency of any numerical model can be regarded as the ability to describe accurately a phenomenon in the shortest possible time. Therefore, we have firstly assessed the quality of our model by statistically comparing estimates with ground measurements. Then, we have confronted these results and the corresponding computation time to those retrieved using other typical models. 


\subsubsection{Model's accuracy: comparison with ground measurements and results from other existing models}

In order to evaluate the accuracy of our model, we have compared horizon estimates with 10 in situ horizons collected during a measurement campaign achieved in Corsica in 2010; both the geographic distribution and the topographic situation of these sites are presented in Figure 7. The availability of an adapted material was one of the main reasons for choosing Corsica, the other one being the existence of many different types of terrain within a small area, which allowed retrieving a significant sample of measurements without difficulties. In order to measure the different horizons, we have used a topographic mapping device, the Leica Builder 100 theodolite (azimuth accuracy =9"; elevation accuracy $=6^{\prime \prime}$ ). The operating principle of this accurate device, mainly used by topographers for measuring terrain slope and aspect, is depicted in Figure 8.

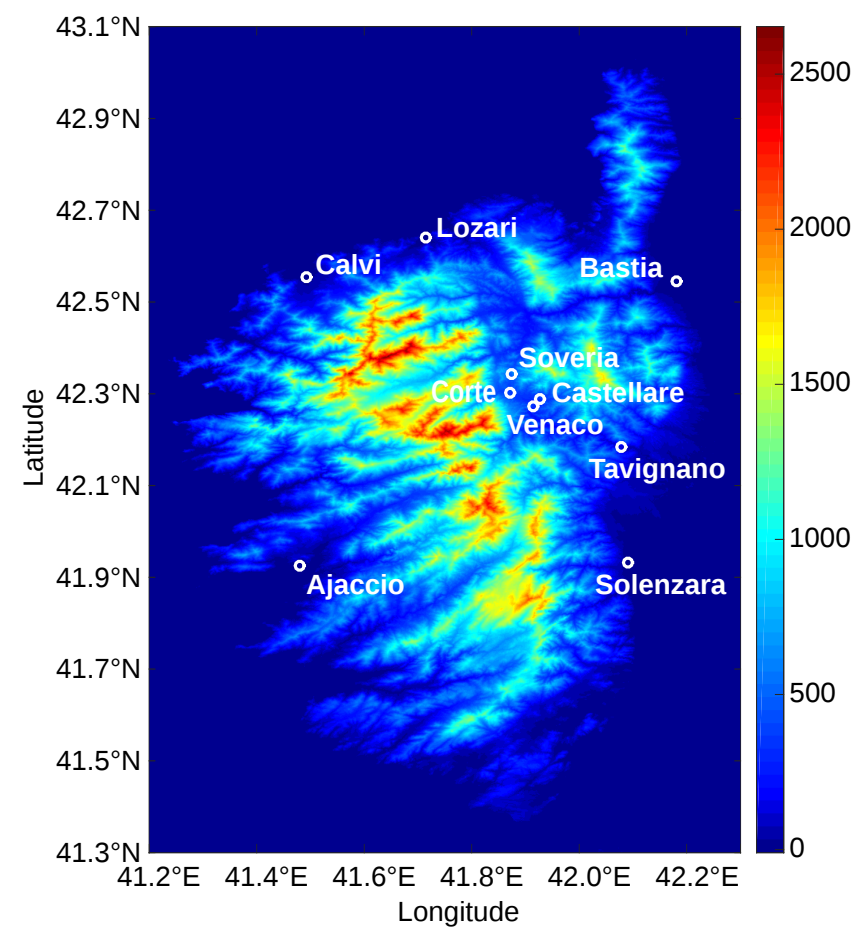

Figure 7: Topographic SRTM-DEM map of Corsica island (meters) with location of the measurement sites.

Furthermore, in order to endorse our model, it was also relevant to compare it to other ones. Thus, we have meanwhile analyzed the results of both the French software Carnaval and the r.horizon add-on of the GRASS GIS open source. Currently released by Sober Software (http: //www. sober-software.com), Carnaval is based on the SRTM-3 DEM and only computes single horizons intended to be used with other solar simulation software. On the contrary, r.horizon is closer to our own model since it is dedicated to the integration of terrain effects into solar potential maps computed by the solar radiation model r.sun (Ruiz-Arias et al., 2009; Š́ri and Hofierka, 2004). Besides, this model allows 2 different computing configurations using a DEM implemented by the user: the first one retrieves shading maps by computing angular elevation for a specific azimuth; the second configuration, and the one we have considered in this work, estimates the full horizon around a given point.

The experiment achieved in 2010 consisted in measuring horizons on 10 different sites across Corsica, representing a full 


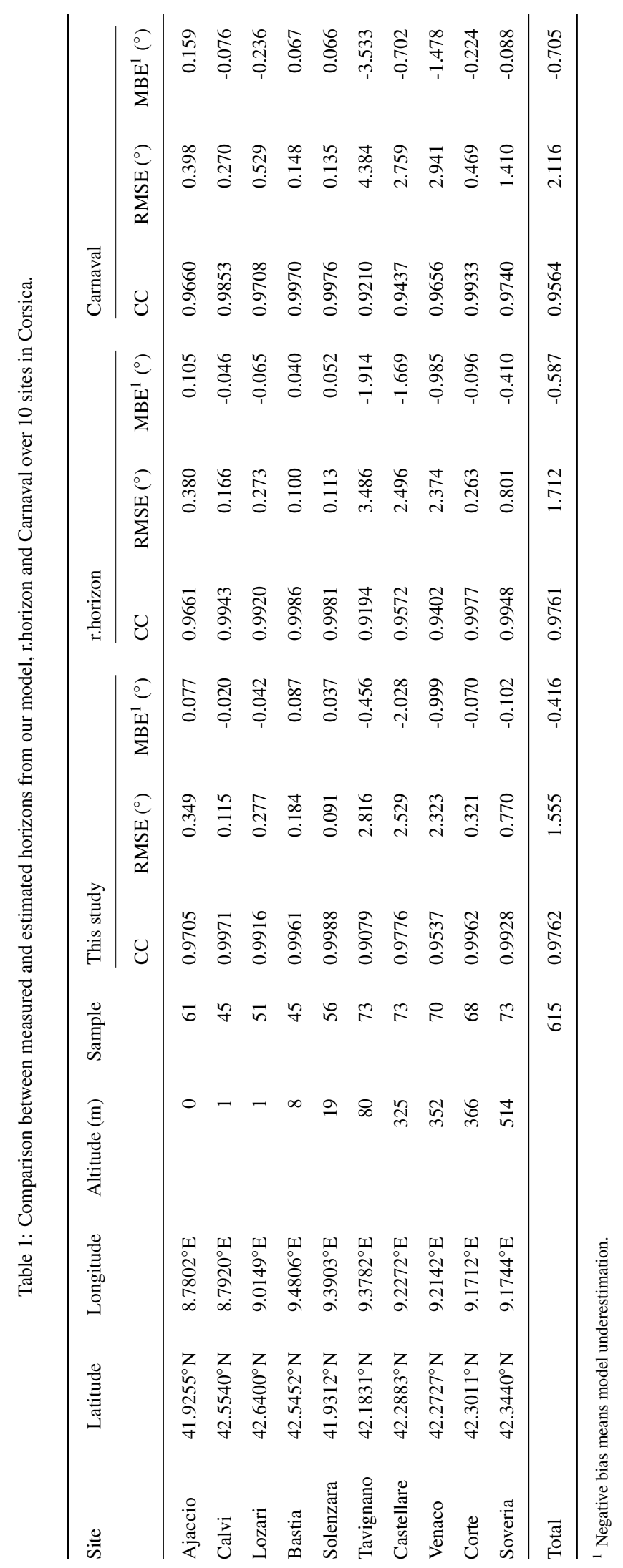




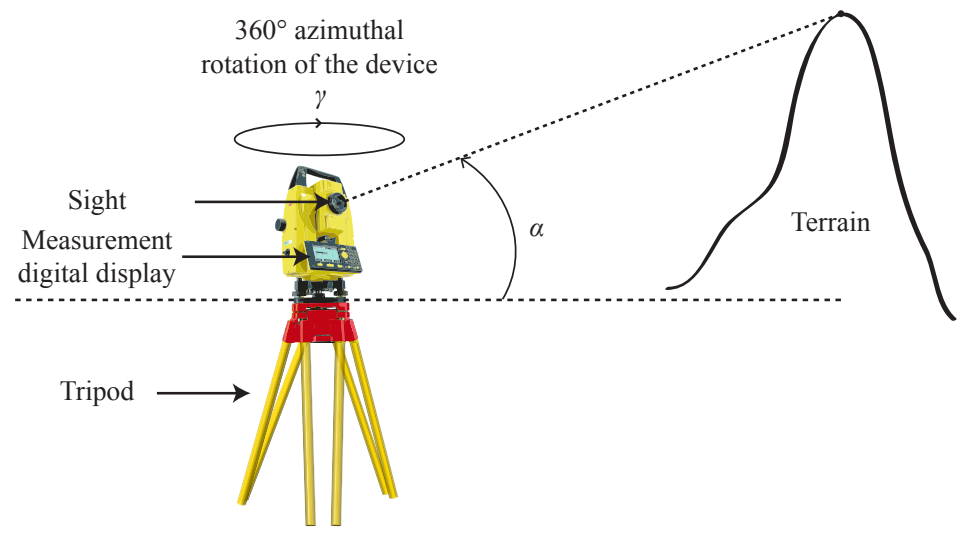

Figure 8: Theodolite operating principle. This device allows both the azimuth $\gamma$ and the angular height $\alpha$ of a given point to be accurately measured.

set of 615 points. Moreover, in order to compare the exact same points from both estimated and measured horizons, we have linearly interpolated modeled horizons, at $1^{\circ}$ azimuth step, over measured azimuths. Then, in order to assess the accuracy of each model, we have considered the following well-known indicators: the root mean square error (RMSE), the mean bias error (MBE) and the correlation coefficient (CC). All final results of the comparison are described in Table 1, where each location is defined by the geographic coordinates and the DEM-based altitude of the given observation point.

Globally, these results show higher accuracy of our model, while Carnaval presents the lowest one. Thus, our model and r.horizon present the most homogeneous correlation for all samples with 2 close correlation coefficients (respectively 0.9762 and 0.9761$)$. Meanwhile, our model also exposes a better RMSE $\left(1.555^{\circ}\right.$ against $\left.1.712^{\circ}\right)$ and a lower negative bias $\left(-0.416^{\circ}\right.$ against $\left.-0.587^{\circ}\right)$. Finally, Carnaval presents the less fitted results regardless of the considered indicator: a correlation coefficient of 0.9564 , a RMSE of $2.116^{\circ}$ and a bias of $-0.705^{\circ}$. Furthermore, we can note that the increase by 10 of the horizon azimuth resolution of our model only reduces RMSE by $0.1 \%$, from $1.555^{\circ}$ to $1.547^{\circ}$, which validates the initial choice of a $1^{\circ}$ azimuth step.

\subsubsection{Model's running time}

By measuring horizons and comparing them with the estimates of other models, we have demonstrated the accurateness and the relevance of the one developed in this study. Most of all, its theoretical basis will allow accurate estimation of any horizon in the world, as long as the SRTM-based DEM exists. Nonetheless, as previously depicted, this model has been primarily designed for considering shading effects within satellite-based solar radiation maps (Bosch et al., 2010; Haurant et al., 2012; Pillot et al., 2013; Ruiz-Arias et al., 2010). Therefore, as with the previous optimization method, used to reduce the area size required to compute all horizons of a given region, everything has been made to minimize computation time.

Firstly, as our model is written with the Matlab programming language, it takes benefit from the software specific matrix calculations, which saves time for handling of large arrays. Thus, on the same machine and for the same viewing distance 
Table 2: Approximate horizon per-thread computation time for a $50 \mathrm{~km}$ view distance around observation point.

\begin{tabular}{|c|c|c|}
\hline Model & Runni & ig time (s) \\
\hline This study & $\approx$ & 0.5 \\
\hline r.horizon ${ }^{1}$ & $\approx$ & 2.0 \\
\hline Carnaval $^{2}$ & $\gg$ & 2.0 \\
\hline
\end{tabular}

$(50 \mathrm{~km})$, Table 2 shows our model is, per thread and per horizon, 4 times faster or more than r.horizon and Carnaval ${ }^{2}$. Besides that, our model is also directly incorporated into the process implemented for considering topography within radiation maps (Pillot, 2014; Pillot et al., 2013), which avoids extra time consumption during data transmission with external models. Finally, our model's source code used to estimate all horizons of a given region is parallelized, which reduces running time as much as the number of available threads within the computer's processor.

\subsection{DEM-based error prediction}

A more detailed reading of Table 1 reveals that, regardless of the given model, error and bias also dramatically vary from one horizon to another. Indeed, some sites present very good estimates (Bastia, Solenzara or Calvi) while some others show both high RMSE and negative bias (Tavignano, Venaco or Castellare). If the original DEM error obviously affects the final quality of the horizon estimates, it is also interesting to know whether this error depends on some specific topographic parameters, which would have influenced the original SRTM precision. In that case, the resulting horizon error would also depend on some specific features; consequently, we propose a method for predicting this error, or at least to detect if the error of any given horizon will probably be significant.

Leaving aside possible measurement errors (angular height, azimuth or geographic coordinates of the observation point), the main idea is to connect the horizon error to the DEM error. To perform this, it is firstly necessary to know the kind of error existing within the SRTM DEM. It is possible to enumerate 2 main errors: vertical and georeferencing errors, respectively achieved on the altitude and the geographic coordinates of a DEM point (Farr et al., 2007; Rodríguez et al., 2006). In this study, we have only considered the vertical error, easier to implement and the one which mainly induces the angular elevation error.

In order to predict the error generated by the DEM quality, it is firstly relevant to express the RMSE of a given horizon

\footnotetext{
${ }^{2}$ So as to get an idea of the computation time over areas, at 2 s/horizon, it would spend about 30 days, 2000 days or 250 years to retrieve all horizons from Corsica, France or Africa respectively.
} 
consisting of $n$ points:

$$
\mathrm{RMSE}=\sqrt{\frac{\sum_{i=1}^{n}\left(\alpha_{i}^{\prime}-\alpha_{i}\right)^{2}}{n}}
$$

With $\alpha_{i}^{\prime}$ the ith estimated angular elevation and $\alpha_{i}$ the ith measured one. Let us then focus on the ith point of this horizon, of angular height $\alpha_{i}$; it is known this point has a vertical error on its DEM altitude $h_{i}^{\prime}$, with respect to the true altitude $h_{i}$, which is propagated on the angular error $\alpha_{i}^{\prime}-\alpha_{i}$. So, if it is possible to predict the vertical error $h_{i}^{\prime}-h_{i}$, it is also possible to assess the resulting angular error, by estimating $\alpha_{i}$ from $h_{i}$ using relation (9). Essentially, we have thus been led by the main idea of looking for an empirical expression, allowing us to merely define the vertical error as a function of SRTM DEM-derived parameters.

Validation of the SRTM has shown the vertical error can be regarded as a random variable following an almost normal probability law, centered on 0 and having a standard deviation specific to each continent (Farr et al., 2007; Rodríguez et al., 2006). Meanwhile, the Miliaresis and Paraschou (2005) and Gorokhovich and Voustianiouk (2006) studies have highlighted the error correlation with topographic features of a given terrain, typically both the slope and aspect of a DEM pixel. In this study, we have only considered the slope $S$, since the aspect is less significant but also more difficult to model as it is highly dependent on the study region. The slope is a function of the altitude gradient to the north $(y)$ and to the east $(x)$ (Zhou and Liu, 2004):

$$
S=\arctan \left(\sqrt{\left(\frac{\partial h}{\partial x}\right)^{2}+\left(\frac{\partial h}{\partial y}\right)^{2}}\right)
$$

Several methods exist for calculating $S$, among which the one developed by Fleming and Hoffer presents a good precision (Jones, 1998). It is based on the formulation of the partial derivatives of the height $h$ (Zhou and Liu, 2004):

$$
\frac{\partial h}{\partial x}=\frac{h_{E}-h_{W}}{2 R_{D E M}^{x}} \quad ; \quad \frac{\partial h}{\partial y}=\frac{h_{N}-h_{S}}{2 R_{D E M}^{y}}
$$

Where $h_{E}, h_{W}, h_{N}, h_{S}$ are respectively heights of eastern, western, northern and southern pixels contiguous to the pixel of interest, $R_{D E M}^{x}$ the DEM metric resolution along $x$ axis, and $R_{D E M}^{y}$ the resolution along $y$ axis. Table 3 summarize, for each site, the main statistical parameters (mean, standard deviation and range) of the horizon slope, that is the slope of all DEM pixels composing the horizon.

Both Miliaresis and Paraschou (2005) and Gorokhovich and Voustianiouk (2006) studies have emphasized the increase of the mean vertical error with the slope $S$ (overestimation of the DEM altitude compared to the true altitude). Miliaresis and Paraschou (2005) especially have shown that, even though the error distribution was translated to the right, this distribution was still close to the one for low slopes $\left(S<2^{\circ}\right)$ with about the same flattening and asymmetry. Considering these assumptions, it is then possible to define the vertical error as:

$$
h^{\prime}-h=r+f(S)
$$


Table 3: Main characteristics of the horizon slope.

\begin{tabular}{lrrl}
\hline Site & \multicolumn{3}{c}{ Slope $\left({ }^{\circ}\right)$} \\
\cline { 2 - 4 } & Mean & Std & \multicolumn{1}{l}{ Range } \\
\hline Ajaccio & 10.1 & 7.4 & $0.0-33.5$ \\
Calvi & 11.1 & 9.4 & $0.6-40.5$ \\
Lozari & 8.7 & 8.7 & $0.0-46.9$ \\
Bastia & 6.4 & 7.8 & $0.4-35.3$ \\
Solenzara & 7.9 & 8.5 & $1.2-39.3$ \\
Tavignano & 21.7 & 5.0 & $9.3-31.1$ \\
Castellare & 16.0 & 6.2 & $4.0-35.8$ \\
Venaco & 14.4 & 6.9 & $0.5-35.8$ \\
Corte & 16.0 & 8.7 & $1.4-39.8$ \\
Soveria & 17.3 & 7.3 & $2.0-38.0$ \\
\hline
\end{tabular}

Where $r$ is a normally distributed random variable centered on 0 such as $r \sim N(0, \sigma)$, resulting of the SRTM validation (Rodríguez et al., 2006), and $f(S)$ any function of the pixel's slope $S$. From the Miliaresis and Paraschou (2005) study, we can then consider, in first approximation, the error linearly increases with the slope. Finally, the expected value of the vertical error $h^{\prime}-h$ is given by:

$$
\mathrm{E}\left[h^{\prime}-h\right]=\mathrm{E}[r]+\beta S=\beta S
$$

Where $\beta$ is a strictly positive constant (distribution translated to the right). Getting $h$ from relation (29), it is then possible to predict the value of the corresponding measured angular elevation $\alpha$, using equation (9). In order to evaluate the constant $\beta$, we have minimized the quadratic error between the measured RMSE of the full sample in Table 1 and the RMSE estimated with this method. Carnaval and r.horizon presenting different errors, the constant $\beta$ is therefore different from one model to another, and is respectively equal to $0.357,0.398$ and 0.494 for our model, r.horizon and Carnaval. In addition, the correlation between the prediction and the measure for each model is given by the correlation coefficient. It is equal, for a $1 \%$ significance level, to $0.9331,0.9611$ and 0.9800 for our model, r.horizon and Carnaval respectively. Finally, the consistency of the prediction method is depicted in Figure 9, with on the one hand the estimates vs measurements scatter plot, and on the other hand the comparison between 2 horizons presenting low (Corte) and high error (Tavignano).

Some aspects of this analysis must be emphasized. First of all, we have not taken into consideration the vertical error of the horizon observation point, although it also interferes in the global angular error. Indeed, if the many points composing the horizon allow smoothing the approximate formula (29), the possible bias introduced by the observation point would, on the contrary, impact all the points, and so could strongly distort the final estimate. This point leads to another aspect of 


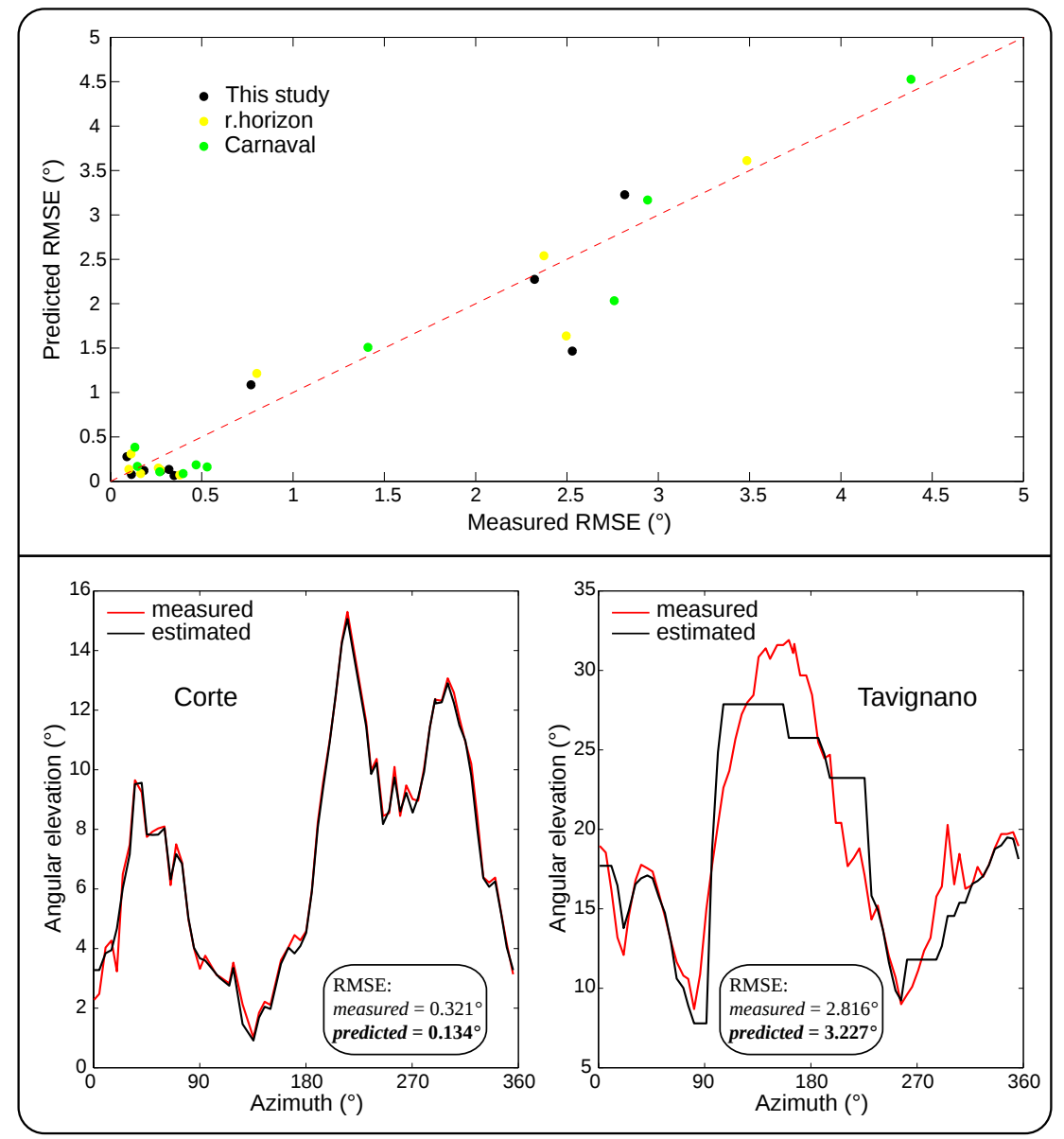

Figure 9: Predicting SRTM-3 DEM-based horizon error. Above is the scatter plot of predicted against measured RMSE values for the 10 horizons depicted in Table 1. Below is an example of horizon measurements and estimates over Corte and Tavignano, respectively for a low and a high error.

this study: the greater the number of points composing the horizon, the more accurate the quadratic error prediction will be. Furthermore, vegetation is also a subject of concern for the error estimation since depending on its type and density, the wavelength of the $\mathrm{C}$ band might penetrate into the canopy (Farr et al., 2007). Accordingly, in vegetated areas, SRTM DEM elevation is located between the ground and the top of the canopy, at a distance which varies significantly with the SRTM DEM variability (Carabajal and Harding, 2006). As the shading effects are influenced by the canopy, this might lead to some extra underestimation, mainly when the horizon is close. Finally, linear approximation between vertical error and slope as well as the decision to leave aside DEM aspect and georeferencing may also explain the shift between measured and predicted values. Even so, this method shows quite accurate estimates, and can further be generalized to all the SRTM-3 DEM, by mainly regarding the predicted RMSE as a sensitivity coefficient allowing the horizon's level of quality to be assessed. 


\subsubsection{Solar irradiation loss} 2012).

As depicted in previous studies, the integration of shading effects improves both the accuracy and spatial information provided by satellite-derived solar radiation maps (Haurant et al., 2012; Pillot, 2014; Ruiz-Arias et al., 2010). However, as described above, the DEM-based horizon computation results itself in some error, which necessarily propagates to the solar irradiation. In order to assess how this error affects the final irradiation estimation, we have used the disaggregation methodology we have developed in another study (Pillot, 2014), coupled with the ESRA clear-sky model developed by Rigollier et al. (2000) and corrected in altitude by Geiger et al. (2002). This methodology includes shading effects into the global solar radiation by applying horizon-derived factors to its diffuse isotropic, diffuse circumsolar and beam components.

Besides, depending on both the sun path and the sky cloudiness, the final influence of the horizon on the solar radiation can be significantly different. In order to apprehend this double dependence, we have estimated the clear-sky daily irradiation throughout the year, and analyzed the influence of the solar radiation's diffuse component by acting on the key parameter of the ESRA model: the Linke turbidity factor $T_{L}$ (Geiger et al., 2002; Rigollier et al., 2000). This coefficient usually varies from 1 to $10, T_{L}=2$ corresponding to a very clear atmosphere, and $T_{L}=8$ corresponding to a polluted atmosphere (Eltbaakh et al.,

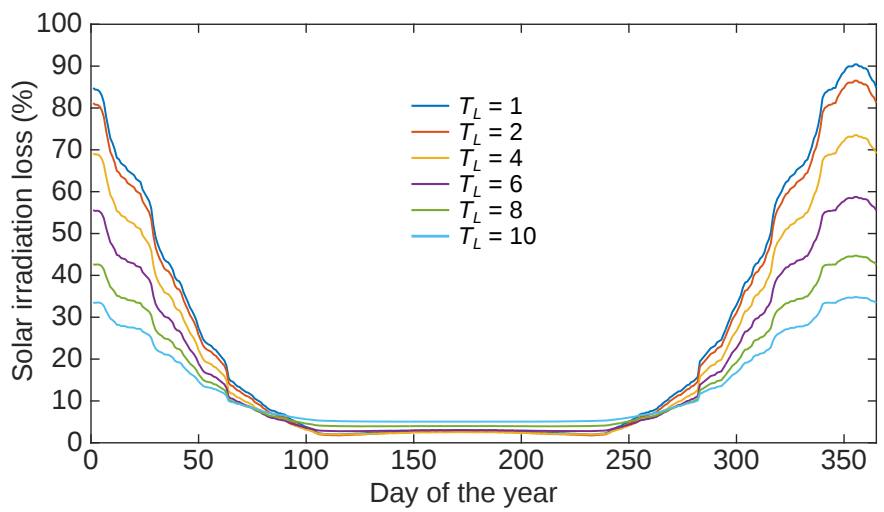

Figure 10: Relative irradiation loss $(\%)$ caused by the horizon at Tavignano site, with respect to the day of the year and the Linke turbidity factor.

In order to evaluate the solar irradiation loss potentially caused by the horizon, we have determined the daily clear-sky irradiation reaching the Tavignano site, with and without shading effects computed from the measured horizon. Figure 10 depicts the final relative loss according to the day of the year and the Linke turbidity factor. As expected (Quaschning and Hanitsch, 1998), shading effects affect more the beam component than the diffuse radiation: the irradiation blocked by the horizon decreases when $T_{L}$ increases. When the sun is high however, between days 100 and 250 , the loss remains low and similar $(<10 \%)$ regardless of the Linke coefficient. In that case, the most significant loss $(>50 \%)$ appears at the end of the autumn and the beginning of the winter, as a consequence of the low sun path. 


\subsubsection{Horizon error propagation}

In order to assess how the RMSE of $1.555^{\circ}$ (Table 1) ends up affecting the solar estimation, as well as the evolution of this influence, we have statistically analyzed the daily irradiation with shading effects reaching the 10 sites of Table 1 , using either the horizon measurements or the estimates from our model. To do this, we have computed the relative root mean square error (RRMSE) between both the results, according to the day of the year and the Linke turbidity factor, depicted in Figure 11. Again, as expected, the beam radiation is more impacted by the horizon shadowing than the diffuse radiation, as the final error decreases with the rise of the Linke coefficient. Also, the RRMSE is significantly variable over the year, compared to the smooth tendency of the solar radiation loss; it can probably be explained by the original variability of the sign of the bias between the estimate and the measured horizon elevation depending on the azimuth. Essentially, in this study, depending on the day and the diffuse fraction, the horizon mismatching results in a final error ranging from approximately $0.5 \%$ (high sun path) to $5.5 \%$ (low sun path) of the daily solar irradiation.

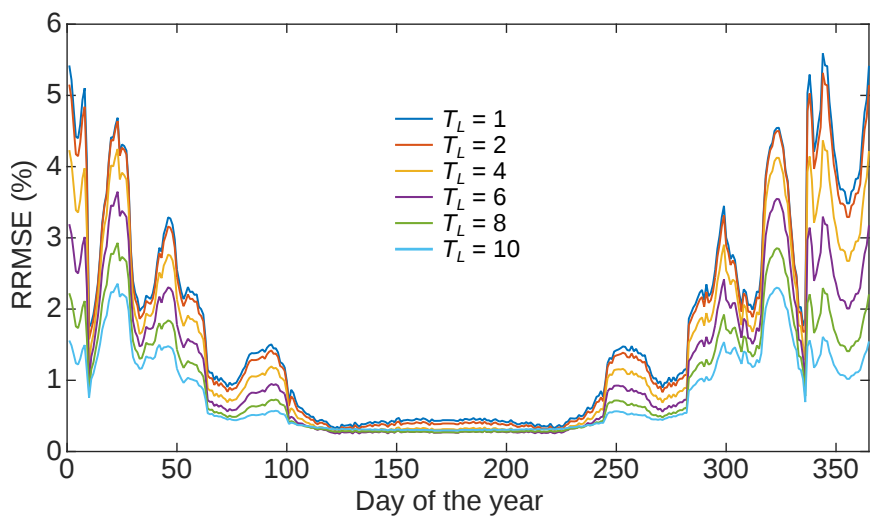

Figure 11: RRMSE (\%) between solar irradiation with shading effects computed from horizon measurements and estimates for all sites of Table 1, with respect to the day of the year and the Linke turbidity factor.

\section{Conclusion}

Many models now retrieve solar radiation from satellite data, but are still limited to an atmospheric characterization without any consideration of the interaction between radiation and Earth's surface. Therefore, in order to improve the accuracy of these satellite-based estimates, some post-processing methods have been proposed for integrating terrain-based effects into final solar irradiance or irradiation maps using DEMs. The main process of all these methods consists in estimating the obstructed horizon around every DEM pixel, in order to evaluate direct and diffuse shadowing. Nevertheless, depending on the size of the study area, computation can also be really time consuming. That is why we have developed and validated a new efficient horizon model, combined with an optimization method and a DEM-based error prediction study.

We have developed this model using the global DEM retrieved from the SRTM, as it is currently the most consistent and accurate available DEM. We have furthermore only based this model on the mathematical statements used to represent the 
DEM, avoiding any region-specific empirical assumption so as to allow the model to be used at any world location with the same expected accuracy. Then, we have assessed its efficiency by comparing its precision and computation time with those of other models. Precision has been retrieved using a statistical comparison between estimates and in situ horizons measured with a theodolite. Finally, we have demonstrated the implementation of our model was relevant, since it is both more accurate (RMSE of $1.555^{\circ}$ against $1.712^{\circ}$ or more for other presented models) and less time consuming (at least 4 times faster than other models).

In addition, we have also proposed an optimization method for reducing horizon computation time in the case of large areas, as well as an empirical approach for predicting the DEM-based error on final estimates. The first one takes into consideration the Earth's roundness in order to limit the maximum zone required to fully describe any horizon of a given region. Hence, it allows knowingly decreasing of modeling accuracy with running time, and thus get the best compromise between them depending on the purpose of the regarded study. The second evaluates the impact of the SRTM DEM original error on the final horizon precision by correlating it to the DEM slope, and has been validated using the in situ horizon sample collected in Corsica.

Finally, we shall note that, in this study, we have not considered the distortion effects of the atmospheric refraction onto the apparent position of objects on Earth, and so on the resulting angular elevation, known as terrestrial refraction (De Graaff Hunter, 1913). This approach seems relevant since, in mapping applications, the additional computation time required for modeling this phenomenon, and its related parameters such as object distance or temperature and pressure conditions, would not necessarily be worth the final accuracy gain. Nevertheless, future research could still improve the accuracy of our current model by focusing on this part.

\section{Acknowledgments}

We are grateful to Dr Michael Mercier-Finidori and the Corsica University Institute of Technology for providing the theodolite we used in the experimental part of this work.

\section{References}

Arvizu, D., Balaya, P., Cabeza, L. F., Hollands, T., Jäger-Waldau, A., Kondo, M., Konseibo, C., Meleshko, V., Stein, W., Tamaura, Y., Xu, H., Zilles, R., 2011. Direct solar energy. In: IPCC special report on renewable energy sources and climate change mitigation. Cambridge University Press, Cambridge, U.K. and New York, NY, USA, Ch. 3.

Ben Ahmed, H., Multon, B., Thiaux, Y., 2011. Consommation d'énergie, ressources énergétiques et place de l'électricité. Techniques de l'ingénieur, Paris, France.

Bermejo, P., Pino, F. J., Rosa, F., Aug. 2010. Solar absorption cooling plant in Seville. Solar Energy 84 (8), 1503-1512.

Beyer, H. G., Costanzo, C., Heinemann, D., 1996. Modifications of the heliosat procedure for irradiance estimates from satellite images. Solar Energy 56 (3), 207 - 212. 
Bosch, J., Batlles, F., Zarzalejo, L., López, G., Dec. 2010. Solar resources estimation combining digital terrain models and satellite images techniques. Renewable Energy 35 (12), 2853-2861.

Bosser, P., 2012. Géométrie de l'ellipsoïde. École nationale des sciences géographiques - Département positionnement terrestre et spatial.

Brisson, A., Le Borgne, P., Marsouin, A., 1999. Development of algorithms for surface solar irradiance retrieval at O\&SI SAF low and mid latitudes. Eumetsat Ocean and Sea Ice SAF internal project team report.

Burkholder, E. F., 2008. Geometrical geodesy. In: The 3-D global spatial data model - Foundation of the spatial data structure. Ch. 6, p. 392.

Carabajal, C. C., Harding, D. J., March 2006. SRTM C-band and ICESat laser altimetry elevation comparisons as a function of tree cover and relief. Photogrammetric Engineering \& Remote Sensing 72 (3), 287 - 298.

De Graaff Hunter, J., 1913. Formulae for atmospheric refraction and their application to terrestrial refraction and geodesy. Survey of India, Dehra Dun, India.

Dozier, J., Frew, J., Sep 1990. Rapid calculation of terrain parameters for radiation modeling from digital elevation data. IEEE Transactions on Geoscience and Remote Sensing 28 (5), 963-969.

Efron, B., 1987. Better bootstrap confidence intervals. Journal of the American Statistical Association 82 (397), 171-185.

Efron, B., Tibshirani, R. J., 1993. An introduction to the bootstrap. Chapman \& Hall, New York, USA.

Eisenhart, L. P., 1909. A treatise on the differential geometry of curves and surfaces. Ginn and Company, Boston, USA.

Eltbaakh, Y. A., Ruslan, M., Alghoul, M., Othman, M., Sopian, K., 2012. Issues concerning atmospheric turbidity indices. Renewable and Sustainable Energy Reviews 16 (8), 6285 - 6294.

Farr, T. G., Rosen, P. A., Caro, E., Crippen, R., Duren, R., Hensley, S., Kobrick, M., Paller, M., Rodriguez, E., Roth, L., Seal, D., Shaffer, S., Shimada, J., Umland, J., Werner, M., Oskin, M., Burbank, D., Alsdorf, D., 2007. The shuttle radar topography mission. Reviews of Geophysics 45 (2).

Geiger, M., Diabaté, L., Ménard, L., Wald, L., 2002. A web service for controlling the quality of measurements of global solar irradiation. Solar Energy 73 (6), 475 - 480.

Gorokhovich, Y., Voustianiouk, A., 2006. Accuracy assessment of the processed SRTM-based elevation data by CGIAR using field data from USA and thailand and its relation to the terrain characteristics. Remote Sensing of Environment 104 (4), 409 $-415$.

Haurant, P., Muselli, M., Pillot, B., Oberti, P., 2012. Disaggregation of satellite derived irradiance maps: Evaluation of the process and application to Corsica. Solar Energy 86 (11), 3168 - 3182. 
Hegerl, G. C., Zwiers, F. W., Braconnot, P., Gillett, N. P., Luo, Y., Marengo Orsini, J. A., Nicholls, N., Penner, J. E., Stott, P. A., 2007. Understanding and attributing climate change. In: Climate change 2007 - the physical science basis. Contribution of working group I to the fourth assessment report of the Intergovernmental Panel on Climate Change. Cambridge University Press, Cambridge, U.K. and New York, NY, USA, Ch. 9.

Hofierka, J., Huld, T., Cebecauer, T., Suri, M., 2007. Open source solar radiation tools for environmental and renewable energy applications. In: Proceedings of the International Symposium on Environmental Software Systems. Prague, Czech Republic.

Janjai, S., Laksanaboonsong, J., Nunez, M., Thongsathitya, A., 2005. Development of a method for generating operational solar radiation maps from satellite data for a tropical environment. Solar Energy 78 (6), $739-751$.

Jarvis, A., Reuter, H., Nelson, A., Guevara, E., 2008. Hole-filled SRTM for the globe version 4. Available from the CGIARCSI SRTM 90m database (http://srtm.csi.cgiar.org).

Jarvis, A., Rubiano, J., Nelson, A., Farrow, A., Mulligan, M., 2004. Practical use of SRTM data in the tropics: comparisons with digital elevation models generated from cartographic data. Tech. rep., Centro Internacional de Agricultura Tropical (CIAT), Cali, Colombia.

Jones, K. H., 1998. A comparison of algorithms used to compute hill slope as a property of the DEM. Computers \& Geosciences 24 (4), $315-323$.

Lemoine, F., Kenyon, S., Factor, J., Trimmer, R., Pavlis, N., Chinn, D., Cox, C., Klosko, S., Luthcke, S., Torrence, M., Wang, Y., Williamson, R., Pavlis, E., Rapp, R., Olson, T. R., 1998. The development of the joint NASA GSFC and the National Imagery and Mapping Agency (NIMA) geopotential model EGM96. Tech. rep., National Aeronautics and Space Administration - Goddard Space Flight Center, Greenbelt, USA.

Liébard, A., De Herde, A., 2005. Aménagement urbain et développement durable en Europe. In: Traité d'architecture et d'urbanisme bioclimatiques. Observ'ER, Paris, France.

Luque, A., Hegedus, S., 2003. Handbook of photovoltaic science and engineering, 1st Edition. John Wiley \& Sons, Chichester, U.K.

Miliaresis, G. C., Paraschou, C. V., 2005. Vertical accuracy of the SRTM DTED level 1 of Crete. International Journal of Applied Earth Observation and Geoinformation 7 (1), 49 - 59.

Mosallat, F., ELMekkawy, T., Friesen, D. L., Molinski, T., Loney, S., Bibeau, E. L., 2013. Modeling, simulation and control of flat panel solar collectors with thermal storage for heating and cooling applications. Procedia Computer Science 19, 686-693.

Muselli, M., Notton, G., Canaletti, J., Louche, A., 1998. Utilization of meteosat satellite-derived radiation data for integration of autonomous photovoltaic solar energy systems in remote areas. Energy Conversion and Management 39 (1), $1-19$. 
NGA, 2000. Department of Defense World Geodetic System 1984 - its definition and relationships with local geodetic systems. Tech. rep., National Geospatial-Intelligence Agency, Springfield, USA.

NGA, 2014. NGA/NASA EGM96, N=M=360 Earth Gravitational Model. http://earth-info.nga.mil/GandG/wgs84/ gravitymod/egm96/egm96.html (access date: 06/01/2015).

Paulescu, M., Paulescu, E., Gravila, P., Badescu, V., 2013. Solar radiation measurements. In: Weather Modeling and Forecasting of PV Systems Operation. Springer, London, U.K., Ch. 2.

Perez, R., Ineichen, P., Moore, K., Kmiecik, M., Chain, C., George, R., Vignola, F., 2002. A new operational model for satellite-derived irradiances: description and validation. Solar Energy 73 (5), 307 - 317.

Perez, R., Seals, R., Zelenka, A., 1997. Comparing satellite remote sensing and ground network measurements for the production of site/time specific irradiance data. Solar Energy 60 (2), 89 - 96.

Pillot, B., 2014. Planification de l'électrification rurale décentralisée en Afrique subsaharienne à l'aide de sources renouvelables d'énergie : le cas de l'énergie photovoltaïque en République de Djibouti. Ph.D. thesis, Université de Corse.

Pillot, B., Muselli, M., Poggi, P., Haurant, P., Hared, I., 2013. The first disaggregated solar atlas of Djibouti: A decisionmaking tool for solar systems integration in the energy scheme. Renewable Energy 57 (0), 57 - 69.

Quaschning, V., Hanitsch, R., 1998. Irradiance calculation on shaded surfaces. Solar Energy 62 (5), 369 - 375.

Rabus, B., Eineder, M., Roth, A., Bamler, R., Feb. 2003. The shuttle radar topography mission - a new class of digital elevation models acquired by spaceborne radar. ISPRS Journal of Photogrammetry and Remote Sensing 57 (4), 241-262.

Rigollier, C., 2004. The method heliosat-2 for deriving shortwave solar radiation from satellite images. Solar Energy 77 (2), 159-169.

Rigollier, C., Bauer, O., Wald, L., 2000. On the clear sky model of the ESRA - european solar radiation atlas - with respect to the heliosat method. Solar Energy 68 (1), 33-48.

Rodríguez, E., Morris, C. S., Belz, J. E., 2006. A global assessment of the SRTM performance. Photogrammetric Engineering \& Remote Sensing (72), 249-260.

Ruiz-Arias, J. A., Cebecauer, T., Tovar-Pescador, J., Šúri, M., 2010. Spatial disaggregation of satellite-derived irradiance using a high-resolution digital elevation model. Solar Energy 84 (9), 1644 - 1657.

Ruiz-Arias, J. A., Tovar-Pescador, J., Pozo-Vazquez, D., Alsamamra, H., Aug. 2009. A comparative analysis of DEM-based models to estimate the solar radiation in mountainous terrain. International Journal of Geographical Information Science 23 (8), 1049-1076. 
Singh, K., Xie, M., 2010. Bootstrap method. In: International Encyclopedia of Education, 3rd Edition. Elsevier, Oxford, U.K., pp. $46-51$.

United Nations, 1998. Kyoto Protocol to the United Nations Framework Convention on Climate Change.

Viebahn, P., Lechon, Y., Trieb, F., 2010. The potential role of concentrated solar power (CSP) in Africa and Europe - a dynamic assessment of technology development, cost development and life cycle inventories until 2050. Energy Policy 39 (8), 4420-4430.

Šúri, M., Hofierka, J., 2004. A new GIS-based solar radiation model and its application to photovoltaic assessments. Transactions in GIS 8 (2), $175-190$.

Walpole, R. E., Myers, R. H., Myers, S. L., Ye, K., 2011. Probability \& statistics for engineers \& scientists, 9th Edition. Pearson, Boston, USA.

Wentzel, M., Pouris, A., 2007. The development impact of solar cookers: a review of solar cooking impact research in south africa. Energy Policy 35 (3), 1909 - 1919.

Zelenka, A., Perez, R., Seals, R., Renné, D., 1999. Effective accuracy of satellite-derived hourly irradiances. Theoretical and Applied Climatology 62 (3-4), 199-207.

Zhou, Q., Liu, X., 2004. Analysis of errors of derived slope and aspect related to DEM data properties. Computers \& Geosciences $30(4), 369-378$. 